\title{
Mini-sternotomy for the Treatment of Aortic Valve Lesions
}

\author{
Altamiro Ribeiro Dias, Ricardo Ribeiro Dias, Fábio Gaiotto, José Lima 0. Júnior, Filinto M.C.N. Cerqueira, \\ Max Grinberg, Roney Sampaio, Paulo de Lara Lavitola, Nelson Elias, Flávio Tarasoutchi, \\ Luiz F. Cardoso, Noedir A. G. Stolf
}

São Paulo, SP - Brazil

Objective - To compare inverted-L mini-sternotomy performed above the sternal furcula with conventional sternotomy in patients with aortic valve diseases who undergo surgical treatment.

Methods - We operated upon 30 patients who had aortic valve lesions that had clinical and hemodynamic findings. All patients underwent inverted-L sternotomy, which extended from above the manubrium of the sternum to the $3^{\text {rd }}$ right intercostal space, without opening the pleural cavity. Their ages ranged from 32 to 76 years, and 18 were males and 12 were females. We used negative pressure in a venous 1/4-inch cannula, and the patients were maintained in Trendelemburg's position. Twenty-seven patients received bioprostheses with diameters ranging from 23 to $29 \mathrm{~mm}$. Three patients underwent only removal of the calcifications of the aortic valve leaflets and aortic commissurotomy.

Results - The mean duration of anoxic cardiac arrest was 63.11min. Access was considered good in all patients. One death was due to pulmonary and renal problems not related to the incision. All patients had a better recovery in the intensive care unit, got out of bed sooner, coughed more easily, and performed prophylactic physiotherapeutic maneuvers for respiratory problems more easily and with less pain in the incision. Early ambulation was more easily carried out by all patients.

Conclusion - Mini-sternotomy proved to be better than the conventional sternotomy because it provided morecomfort for the patients in the early postoperative period, with less pain and greater desire for early ambulation and all its inherent advantages.

Key words: aortic valve disease, aortic valve surgery, sternotomy

Instituto do Coração do Hospital das Clínicas - FMUSP

Mailing address: Altamiro Ribeiro Dias - InCor - Av. Dr. Enéas C. Aguiar, $44-$ 05403-900 - São Paulo, SP, Brazil - aribeirodias@ cardiol.br

English version by Stela Maris C. e Gandour
Interest in using minimally invasive procedures in cardiac surgery has increased greatly around the world.

Lesions in the aortic valve have been approached with this type of procedure with good early results, even though controversies still exist.

Cosgrove and Sabik ${ }^{1}$ reported their experience with right parasternal mini-incisions at the $2^{\text {nd }}, 3^{\text {rd }}$, and $4^{\text {th }}$ costal cartilages to provide access for the surgical treatment of aortic valve diseases.

Mulinari et $\mathrm{al}^{2}$ and Pereira et $\mathrm{al}^{3}$ favor the median access via, such as the reduced sternotomy, for the successful treatment of the lesions of the aortic valve.

Cohn and Gundry ${ }^{4}$ reported good results obtained with the technique of the Cleveland Clinic cited above.

Denton Cooley, on the same occasion, reported having seen some cases of pulmonary hernias resulting from sectioning the costal cartilages. To avoid this problem, Steven Gundry, from Loma Linda, proposed access through mini-sternotomy, with an incision extending from the manubrium of the sternum to the $3^{\text {rd }}$ intercostal space, where the incision is deviated to the right, like an inverted L, preserving the right internal thoracic artery ${ }^{4}$.

Dias et al ${ }^{5}$ reported an initial experience with mini-sternotomy, extending the incision to the $4^{\text {th }}$ intercostal space.

All these controversial approaches resulted in a panel discussion ${ }^{6}$ in which Steven Gundry enthusiastically supported this access approach. On the other hand, Smith ${ }^{6} \mathrm{cri}^{-}$ ticized its indiscriminate use.

Our objective is to report the initial results obtained at the Instituto do Coração of the Hospital das Clínicas of the Medical School of the University of São Paulo with ministernotomy up to the $3^{\text {rd }}$ right intercostal space.

\section{Methods}

We studied 30 patients with aortic valve disease with clinical and hemodynamic repercussion, who were operated upon from June ' 97 to December ' 99 . Their ages ranged from 32 to 76 (mean of 58.72) years. Eighteen patients were males and 12 were females. 
Twenty-two patients had severe dyspnea, 15 had angina, and 5 had subjective complaints compatible with arrhythmias.

Preoperative diagnoses were as follows: aortic stenosis in 16 patients, double aortic valve dysfunction in 7 , and aortic insufficiency in 7.

All patients underwent inverted-L mini-sternotomy, the skin incision extending from the manubrium of the sternum to the $3^{\text {rd }}$ right intercostal space, for 10 to $11 \mathrm{~cm}$ (fig. 1). Opening of the right pleural cavity or lesion of the right internal thoracic artery, or both, did not occur when the transversal segment of the sternotomy was completed.

Trendelemburg's position was adopted in all cases, which facilitated the marsupialization of the heart with fixation and traction of the pericardium to the edges of the mini-incision.

Arterial cannulation was easily performed with a purse-string suture in the ascending aorta, close to the innominate vein. Venous cannulation was performed through a single $1 / 4$-inch wire cannula introduced into the right atrial auricle through a purse-string suture. The use of negative pressure in the venous line made perfect venous drainage possible, facilitating the exposure of the structures (fig. 2). Aspiration of the left cavities was performed with the aid of an aspirator introduced into the left atrium through a pursestring suture in the right superior pulmonary vein in most cases. In 5 patients, very good aspiration was obtained by introducing the aspirator into the pulmonary artery.

All patients underwent surgery with hypothermia at $28^{\circ} \mathrm{C}$ and cardioplegia with St. Thomas solution.

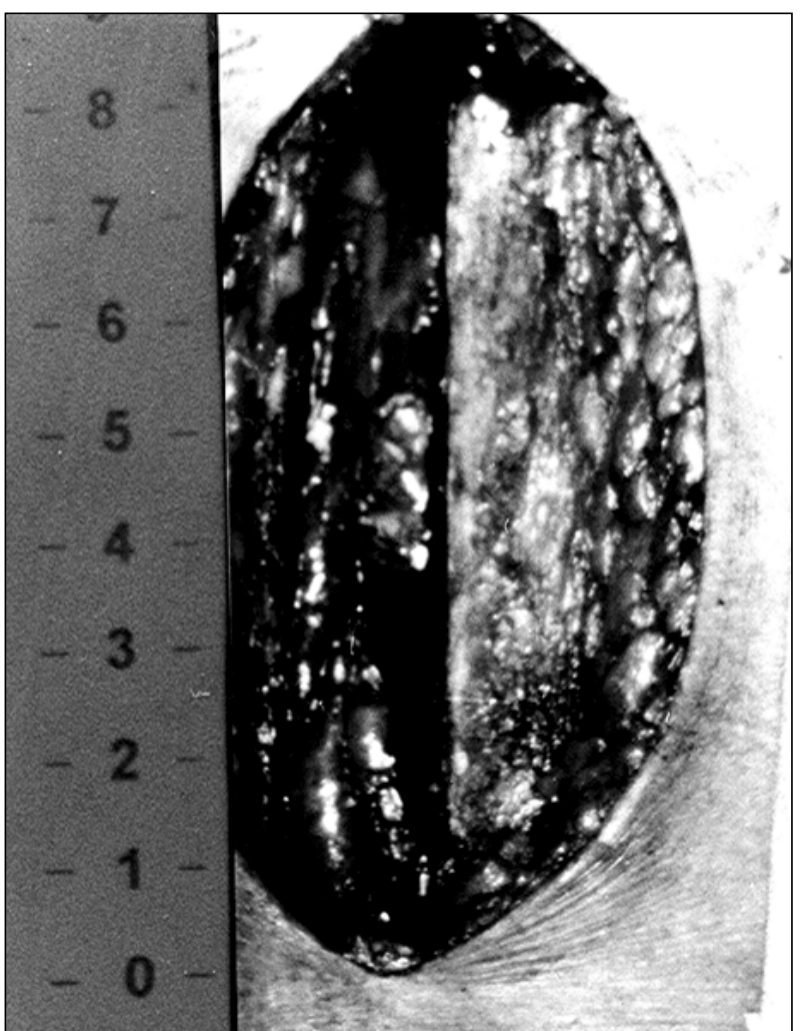

Fig. 1 - Intraoperative photography showing skin incision of approximately $10 \mathrm{~cm}$.

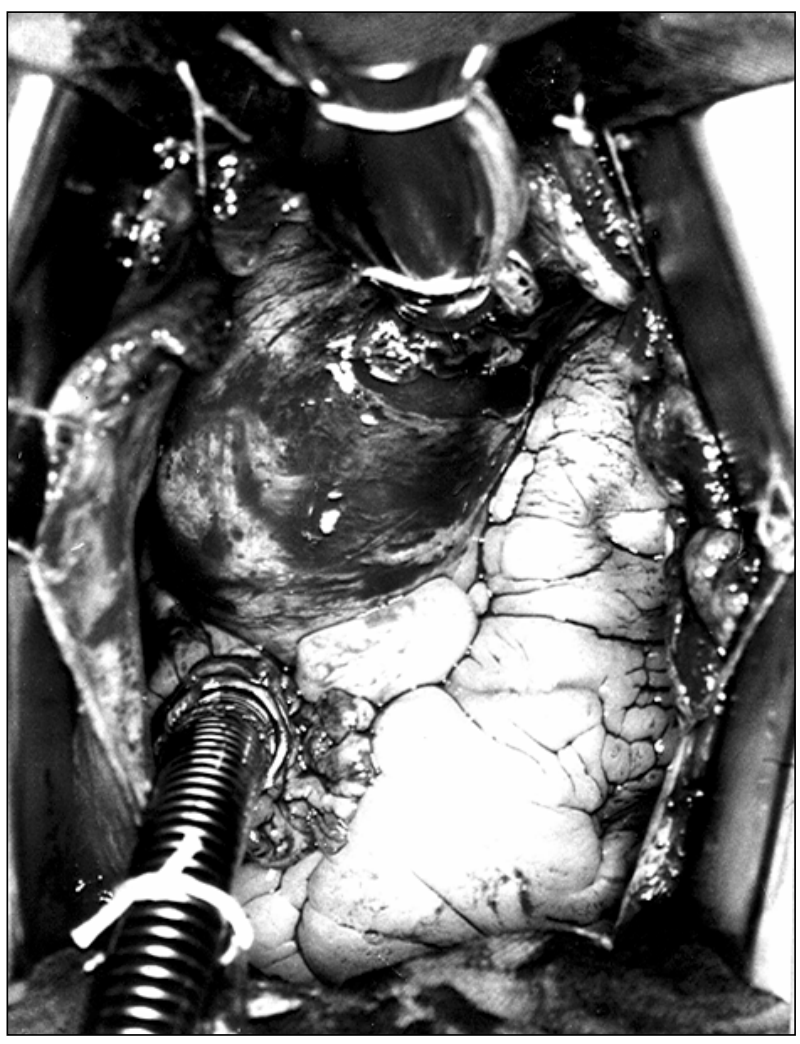

Fig. 2 - Intraoperative photography showing aorta and right atrium cannulated and ready for perfusion. Note the very good exposure of the ascending aorta.

Removal of calcifications on the leaflets of the aortic valve and section of the fusions between these leaflets were possible in 3 patients, with good results on direct visualization and intraoperative echocardiographic assessment (fig. 3).

The remaining patients underwent valvar replacement with removal of the annular calcifications when present. One patient received a bioprosthesis $21 \mathrm{~mm}$ in diameter, 7 patients each received bioprostheses of $23 \mathrm{~mm}, 9$ patients each received bioprostheses of $25 \mathrm{~mm}, 7$ patients each received bioprostheses of $27 \mathrm{~mm}$, and 3 patients each received bioprostheses of $29 \mathrm{~mm}$ of diameter (fig. 4 ).

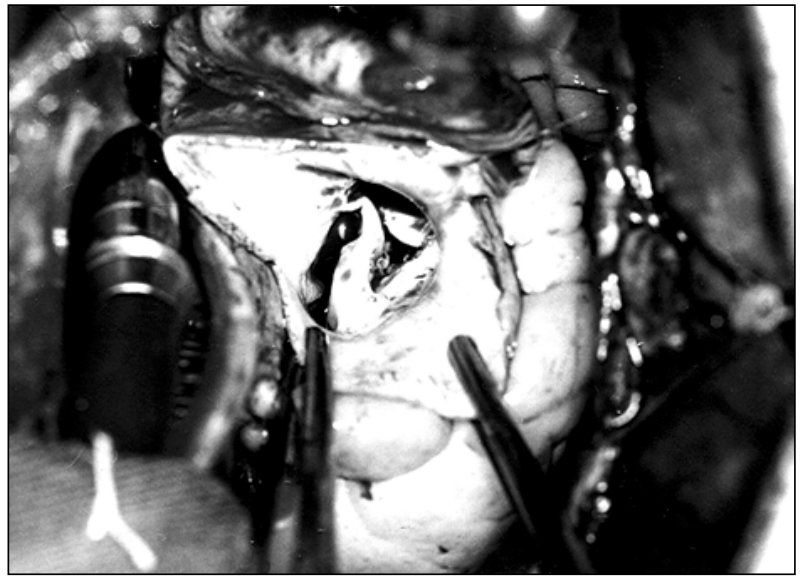

Fig. 3-Once aortotomy has been performed, the fused commissures may be seen in 1 of the 3 patients who underwent aortic commissurotomy. 


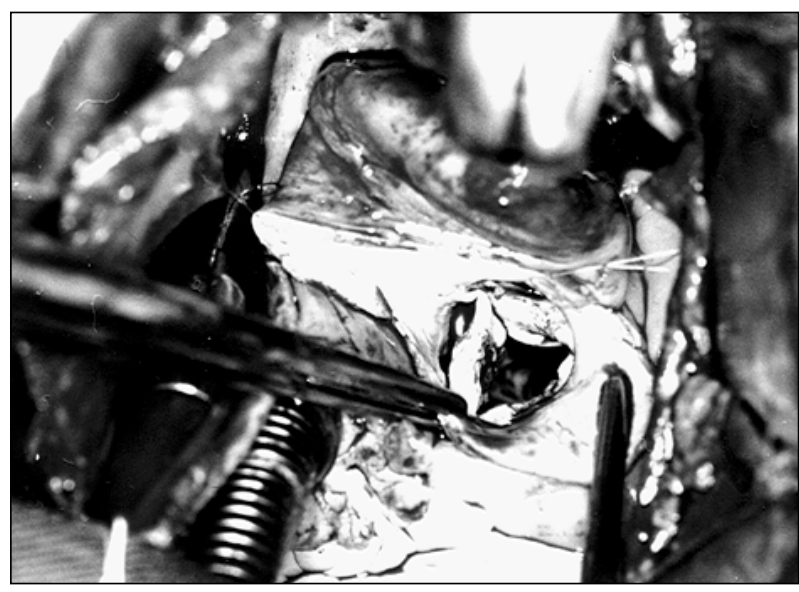

Fig. 4-Note the complete commissurotomy

The management of patients with pure aortic insufficiency was much easier than that of patients with intensely calcified aortic stenosis.

An important point is mediastinal drainage, which should be performed immediately before the end of the anoxic cardiac arrest, prior to reestablishment of cardiac beats. A small incision in the aponeurosis, right below the sternal furcula, really facilitates the introduction of a retrosternal clamp up to the end of the mini-sternotomy to provide fixation of the drain and its exteriorization below the sternal furcula. The still empty cardiac cavities make this procedure easy and safe.

Sternal closing was performed with stitches of steel thread only on the manubrium, no stitch being required for the transversal segment of the sternotomy. The patients were referred to the postoperative unit at the end of the procedure.

\section{Results}

The duration of the anesthesia ranged from 225 to $480 \mathrm{~min}$ (mean of $327.22 \mathrm{~min}$ ), the duration of extracorporeal circulation ranged from 53 to $140 \mathrm{~min}$ (mean of $90.38 \mathrm{~min}$ ), and the duration of the anoxic cardiac arrest ranged from 37 to $103 \mathrm{~min}$ (mean of $63.11 \mathrm{~min}$ ).

No significant bleeding occurred in this group of patients.

It is worth noting that, after regaining consciousness, the patients had greater mobility and greater ability to perform their physiotherapeutic exercises proper to this phase of the postoperative period.

The length of stay in the intensive care unit ranged from 24 to $48 \mathrm{~h}$, and the time in the ward before hospital discharge was around 6 to 7 days.

One patient died due to renal and pulmonary problems not related to the incision.

In our series, ambulation occurred much sooner and was performed more easily and with more willingness compared with that in patients undergoing conventional sternotomy.

After hospital discharge, the patients have been periodically followed up in the cardiac valve outpatient care unit, all of them being in functional class I.

\section{Discussion}

Our initial experience shows that the mini-sternotomy technique is safe, perfectly feasible, and relatively easy to perform. When comparing inverted-L mini-sternotomy with right paramedian vertical thoracotomy, proposed by some authors ${ }^{1,4}$, we can see that the incision above the manubrium of the sternum is more benign and freer from pleuropulmonary complications.

We do not consider it necessary to extend the incision as far as the $4^{\text {th }}$ intercostal space ${ }^{5}$, because the $3^{\text {rd }}$ intercostal space provides enough room for the creation of the pouch in the right atrial auricle, as well as for drainage of the left chambers through a catheter introduced into the superior pulmonary vein.

Caution, however, is required because this access via should not be considered of unlimited application. We emphasize that in short and obese patients, the use of this technique may not be recommended.

In addition to the cosmetic benefit of the small skin incision $(10 \mathrm{~cm})$, the greatest benefit is the comfort experienced by the patients as soon as they recover from anesthesia. The firmness provided by the integrity of the inferior $2 / 3$ of the sternum makes it possible for the patients to cough more easily, to perform their physiotherapeutic exercises more comfortably, and to ambulate sooner with minimum pain in the incision. InCor is an institution affiliated with a university, has well-defined postoperative protocols for conventional procedures, and we did not find it necessary to make any change in these protocols in this initial phase of the procedure. Therefore, no substantial reduction existed in the periods of stay in the intensive care unit or in the period of hospitalization as a whole.

The durations of anesthesia, of perfusion, and of anoxic cardiac arrest also did not differ from those of conventional surgery.

We think that greater experience with and wider application of this type of surgical approach may certainly reduce the duration of hospital stay and the stay in the intensive care unit, with obvious advantages to the patients.

Simple maneuvers, such as those reported, make the procedure easier, provide greater safety, and facilitate its application. Among these maneuvers, we highlight the wide marsupialization of the heart, the venous drainage with a thinner wire cannula, Trendelemburg's position, and aspiration through the pulmonary artery, when necessary.

In our environment ${ }^{2,3}$, this incision and its variants have been used with good results, as has been the case at other international services ${ }^{6}$.

In conclusion, we consider inverted-L mini-sternotomy a safe and efficient approach that enhances the recovery of patients in the early postoperative period.

Late results still require further assessment. However, it is very likely that they will be linked to a lower incidence of problems related to the incision, with no direct interference with heart disease. 


\section{References}

1. Cosgrove DM, Sabik JF. Minimally invasive approach for aortic valve operations. Ann. Thorac Surg 1996; 62: 596-7.

2. Mulinari LA, Tyszka AL, CostaF, et al. Miniesternotomia. Um acesso seguro para cirurgia cardíaca. Anais do $24^{\circ}$ Congresso Nacional de Cirurgia Cardíaca. Campo Grande, MS 1997: 95.

3. Pereira WM, Frota Filho JD, Jung LA, et al. Acesso restrito para cirurgia da valva aórtica (Minimamente invasiva) Anais do $24^{\circ}$ Congresso Nacional de Cirurgia Cardíaca, Campo Grande, MS, 1997: 115.
4. Cohn L, Gundry SR. Adult cardiac symposium. $77^{\text {th }}$ Annual Meeting of the American Association for Thoracic Surgery, p.6-8. Washington, 4 to 7 may, 1997.

5. Dias RR, Avelar Júnior SF, Lima SGG, et al. Miniesternotomia para a cirurgia da valva aórtica: experiência inicial. Rev Bras Cir Cardiovasc 1998; 14: 317-20.

6. Kouchoukos NT, Gundry SR, Smith CR. Controversies in Cardio Thoracic Surgery. Acquired cardiac controversies. Aortic valve replacement should be performed through a mini- sternotomy $80^{\text {th }}$ annual Meeting. American Association for Thoracic Surgery. Abril 30 - May 3.2000-07-17 Toronto. Ontario, Canadá. 